(C) 2016 IEEE. Personal use of this material is permitted. Permission from IEEE must be obtained for all other uses, in any current or future media, including reprinting/republishing this material for advertising or promotional purposes, creating new collective works, for resale or redistribution to servers or lists, or reuse of any copyrighted component of this work in other works. 


\title{
EEG-based Driver Fatigue Detection using Hybrid Deep Generic Model*
}

\author{
Phyo Phyo $\operatorname{San}^{2}$,Sai Ho Ling ${ }^{1}$, Rifai Chai ${ }^{1}$, Yvonne Tran ${ }^{1,3}$, Ashley Craig ${ }^{3}$, and Hung Nguyen ${ }^{1}$
}

\begin{abstract}
Classification of electroencephalography (EEG)based application is one of the important process for biomedical engineering. Driver fatigue is a major case of traffic accidents worldwide and considered as a significant problem in recent decades. In this paper, a hybrid deep generic model (DGM)based support vector machine is proposed for accurate detection of driver fatigue. Traditionally, a probabilistic DGM with deep architecture is quite good at learning invariant features, but it is not always optimal for classification due to its trainable parameters are in the middle layer. Alternatively, Support Vector Machine (SVM) itself is unable to learn complicated invariance, but produces good decision surface when applied to well-behaved features. Consolidating unsupervised high-level feature extraction techniques, DGM and SVM classification makes the integrated framework stronger and enhance mutually in feature extraction and classification. The experimental results showed that the proposed $\mathrm{DBN}$-based driver fatigue monitoring system achieves better testing accuracy of 73.29 $\%$ with $\mathbf{9 1 . 1 0} \%$ sensitivity and $\mathbf{5 5 . 4 8} \%$ specificity. In short, the proposed hybrid DGM-based SVM is an effective method for the detection of driver fatigue in EEG.
\end{abstract}

\section{INTRODUCTION}

Fatigue is one of the key factor in road accidents for driver in transportation [1]. Driver fatigue has been described as a feeling of tiredness and reduced alertness when driving which is associated with drowsiness, and which impairs capability and willingness to perform the driving task [2]. The symptoms of driver fatigue include increased feelings of tiredness, slower reaction time and lack of concentration during driving and reduced control of speed of the vehicle [3].

Recently, physiological measurement is considered one of an effective measurement on driver fatigue. Electroencephalography (EEG) [4], [5], electrooculography (EOG) [6], and electrocardiography (ECG) [7] associated with fatigue were investigated. EEG is considered to be a significant and reliable method of detecting fatigue, as it directly measures neurophysiological activity in the human brain [4]. Also, EEG has been used frequently for sleep research [8]. Accordingly, this paper explores strategies for improving the fatigue classification in an EEG-based system by using advanced computational intelligence.

\footnotetext{
$1 *$ Sai Ho Ling, Rifai Chai, Yyonne Tran, and Hung Nguyen are with Center for Health Technologies, Faculty of Engineering and IT, University of Technology Sydney, Australia Steve.Ling, Rifai Chai, Hung. Nguyenduts . edu . au

${ }^{2}$ Phyo Phyo San, is with the Data Analytic Department, Institute for Infocomm Research, Singapore, sanppei2r.a-star.edu.sg

${ }^{3}$ Ashley Craig is with the Rehabilitation Studies Unit, Sydney Medical School, The University of Sydney, NSW, Australia, a.craig@sydney.edu.au
}

Recently, deep belief networks (DBNs) have been successfully applied in various domains, raining from image classification [9], speech recognition, audio classification [10] [11] to natural language processing [12]. Besides the successful stories of image and speech recognition, these approaches have been introduced in physiological signals such as electromyogram (EMG), electrocardiogram (ECG), electroencephalogram (EEG) signals [13] due to their merits in high-level feature representation and better classification performance. In all the above mentioned applications, the deep learning models employed feed-forward neural network (FFNN) model as well as softmax activation function (also known as multinomial logistic regression) for final fine-tuning and classifications. As an alternative to deep framework with neural network structure, support vector machine (SVM) has been introduced to train on high-level features extracted by the final layer of restricted boltzmann machine (RBM) [14]. In particular, deep structure of RBM are trained to learn good invariant hidden layer representation while the corresponding hidden variables of data samples are treated as inputs to SVM. This recent surge of activities are largely spurred its use in the detection of premature ventricular contraction (PVC) in ECG signal. To the best of our knowledge, the deep learning framework have not been applied yet.

In this paper, considering high-dimensional, non-linear nature of EEG data, a hybrid deep generic model-based SVM (DGM-SVM) classifier is developed for driver fatigue detection. The inputs in this strategy are raw high dimensional EEG data while the output represent fatigue or nonfatigue status. With the proposed integrated system, highlevel EEG features are preliminarily extracted through the layer-wise training of deep hicherical RBM, followed by SVM for classification on the features extracted by RBM. Consolidating the unsupervised feature extraction and SVM classification which makes the integrated frame work to stronger and mutually enhances both features extraction and classification process.

The organization of this paper is as follows: in Section II, an integrated framework, deep generic model-based SVM and its application in EEG-based driver fatigue detection is introduced. To show the effectiveness of our proposed methods, the experimental analysis are compared and analyzed in Section III before a conclusion is drawn in Section IV. 


\section{METHODS}

\section{A. EEG-based fatigue monitoring}

The system block diagram of the driver fatigue monitoring/classification is shown in Fig. 1. The process begun with the EEG data collection of the driving fatigue study using the driving simulation software. The raw EEG signal which contaminated with the artifacts such as ocular, muscular and heart activities are removed. The cleaned EEG data is fed into the next process which is the segmentation with certain duration of windows. After the segmentation, the cleaned raw EEG data is directly feed to the hybrid DGMbased SVM system without the process of feature extraction. In the proposed driver fatigue detection system in Fig. 1, the DGM is used to extract high-level features while SVM is used final classification. Integrating unsupervised feature extraction and SVM classification makes the proposed hybrid frame work stronger and improve the performances in both feature extraction and classification. For comparison purpose,

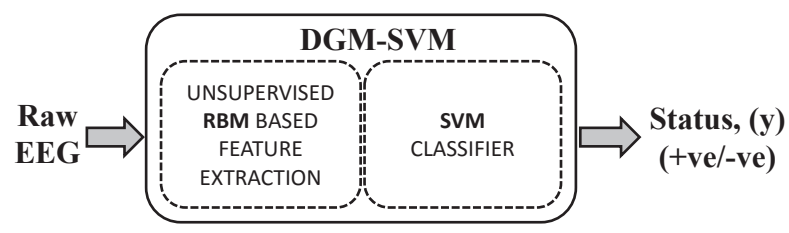

Fig. 1. System block diagram of driver fatigue monitoring system

the standard feature extraction process using power spectrum density (PSD) is introduced and the features extracted by DGM as well as PSD are analyzed in order to see the effectiveness of proposed method in fatigue monitoring system.

\section{B. Hybrid Deep Generic Model with Support Vector Ma- chine (DGM-SVM)}

In this paper, an integrated framework which uses deep generic model and SVM is proposed. In general, a probabilistic generative model with deep architecture is quite good at learning invariant features, but not always optimal for classification since most of the trainable parameters are in the middle layer [15]. On the other hand, SVM itself is unable to learn complicated invariance, while it achieves good decision surfaces on well-behaved features. To overcome individual limitations of DBN and SVM, an integrated system in Fig. 2 is proposed. In the proposed system, a deep generic model is initially constructed by stacking predefined number of restricted boltzmann machines (RBM). In each RBM (for example $R B M^{1}$ in Fig. 2), it consists of visible $m$ visible neuron $\left(v=\left(v_{1}, \ldots, v_{m}\right)\right)$ and $n$ hidden neurons $\left(h=\left(h_{1}, \ldots, h_{n}\right)\right)$. They are fully connected via symmetric undirected weights $w_{i j}$. With the weights and biases, the energy of a joint configuration between hidden and visible neurons, $E(v, h)$ is defined as [16]:

$$
E(v, h)=-\sum_{i=1}^{n} \sum_{j=1}^{m} w_{i j} h_{i} v_{j}-\sum_{j=1}^{m} b_{j} v_{j}-\sum_{i=1}^{n} c_{i} h_{i}
$$

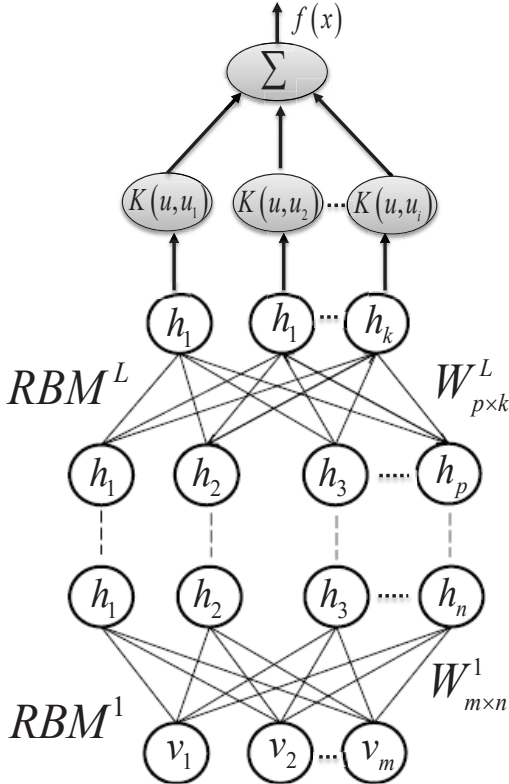

Fig. 2. Hybrid Deep Generic Model-based SVM System (DGM-SVM)

where $w_{i j}$ is associated weight between $v_{j}$ and $h_{i}$ for all $i \in\{1, \ldots, n\}$ and $j \in\{1, \ldots, m\} ; b_{j}$ and $c_{i}$ are bias terms associated with the $j^{\text {th }}$ and $i^{\text {th }}$ visible and hidden neurons. Through the energy function in (1), for all possible pair of visible $(v)$-hidden $(h)$ neurons, the networks assigns a joint probability distribution as follows:

$$
p(v, h)=\frac{e^{-E(v, h)}}{Z}
$$

where $Z=\sum_{v, h} e^{-E(v, h)}$ is the normalized term which is obtained by summing over the energy of all possible $(v, h)$ configurations. Through the gradient of log probability of a visible vector $(v)$ over the weight $w_{i j}$, the updated rule is calculated by constructive divergence (CD) algorithm as follows:

$$
\Delta w_{i j}=\gamma\left(\left\langle v_{j} h_{i}\right\rangle_{\text {data }}-\left\langle v_{j} h_{i}\right\rangle_{\text {recon }}\right)
$$

where $\gamma$ is the learning rate, $v$ and $h$ are visible and hidden units, $\left\langle v_{j} h_{i}\right\rangle_{\text {recon }}$ is the reconstruction of original visible units which is calculated by setting the visible unit to random training vector. Due to the constraints in hidden to hidden connections and visible to visible connections, the binary state of hidden unit and visible unit are considered as follows:

$$
\begin{aligned}
& p\left(h_{i}=1 \mid v\right)=\sigma\left(\sum_{j=1}^{m} w_{i j} v_{j}+c_{i}\right) \\
& p\left(v_{j}=1 \mid h\right)=\sigma\left(\sum_{j=1}^{n} w_{i j} h_{i}+b_{j}\right)
\end{aligned}
$$

where $\sigma$ is the logistic sigmoid function and computed as $\sigma(x)=1 /(1+\exp (-x))$. 
The joint distribution between visible layer and $L$ hidden layer is modeled by:

$$
P\left(v, h^{1}, \ldots, h^{L}\right)=\left(\prod_{k=0}^{L-2} P\left(h^{k} \mid h^{k+1}\right)\right) P\left(h^{L-1}, h^{L}\right)
$$

where $v=h^{0}, p\left(h^{k-1} \mid h^{k}\right)$ is a conditional distribution for the visible units conditioned on the hidden units of the RBM at level $k$, and $P\left(h^{L-1}, h^{L}\right)$ is the visible-hidden joint distribution in the top-level RBM. In order to extract a deep hierarchical features of the input (training) data, the DBN is trained in a greedy layer-wise manner. During the training, each RBM are applied recursively with the features activations by one RBM and represented as the data for training the next RBM in the stack. With the use of optimized bottom up recognition weights of deep hierarchical models, high-level feature set $(u)$ is extracted by:

$$
u=W \times v, \quad W=\left[W^{1}, W^{2}, \ldots, W^{L}\right]
$$

Once the high-level features are extracted, it is integrated to SVM to work as a final classifier. The integrated system in Fig. 2 is quite appealing because feature extraction process is introduced and integrated in a regular SVM kernel $\left(K\left(u, u_{i}\right)\right)$ [17]. Finally, the output of DGM-SVM, $f(x)$ is calculated by:

$$
f(x)=\operatorname{sgn}\left(\sum_{i} y_{i} \alpha_{i} K\left(u, u_{i}\right)+b\right)
$$

where $s g n$ is a signum function, $K\left(u, u_{i}\right)$ is a polynomial kernel function which measures the similarity between input pattern $u$ and the training sample $u_{i}, \alpha$ is the weight parameter for each corresponding input $h_{i}$. The output $f(x)$ computes the discriminant function as a liner combination of the similarity scores with learned weights $\alpha_{i}$. In this clinical application, the output of DGM-SVM $f(x)$ is calculated by (8) and it is defined as positive (fatigue state) when the $f(x)$ is greater than 0 . It can be presented as:

$$
y=\left\{\begin{array}{cc}
+1, & f(x) \geq 0 \\
-1, & f(x)<0 .
\end{array}\right.
$$

\section{Performance Measurement}

To determine the performance of proposed detection system, sensitivity, specificity, and accuracy are introduced [18]:

$$
\begin{aligned}
\operatorname{Sen}(\xi) & =\frac{N_{T P}}{N_{T P}+N_{F N}} \\
\operatorname{Spec}(\eta) & =\frac{N_{T N}}{N_{T N}+N_{F P}} \\
\operatorname{Acc}(\alpha) & =\frac{N_{T P}+N_{T N}}{N_{T P}+N_{T N}+N_{F P}+N_{F N}}
\end{aligned}
$$

where $N_{T P}$ is defined as number of true positive, $N_{F N}$ is number of false negative, $N_{F P}$ is number of false positive, and $N_{T N}$ is number of true negative. The values of these are within 0 to 1 . In clinical study, the accuracy of a given classifier is important because it mainly represents the performance of classifier. The higher accuracy represents the better performance proposed detection system.

\section{RESULT AND DISCUSSION}

In this study, the EEG data from previous study [4] was used which contains of 5 healthy participants aged between 18 and 55 years for driving simulator task using the divided attention steering simulator. During the driving experiment, participants were asked to drive at the center. This driving experiment was stopped with conditions as follows: (i) if signs of fatigue were detected; (ii) if there were off the road driving detected for more than 15 seconds; (iii) if maximum time of 2 hours has been reached.

During this experiment, EEG brain signals were recorded with 32-channels based on the International 10-20 system attached to the head. These 32-EEG channels location are: FP1, AF3, F7, F3, FC1, FC5, T7, C3, CP1, CP5, P7, P3, PZ, PO3, O1, OZ, O2, PO4, P4, P8, CP6, CP2, C4, T8, FC6, FC2, F4, F8, AF4, FP2, FZ and CZ with sampling rate of $256 \mathrm{~Hz}$. The EEG recorded signals is shown in Fig. 3.

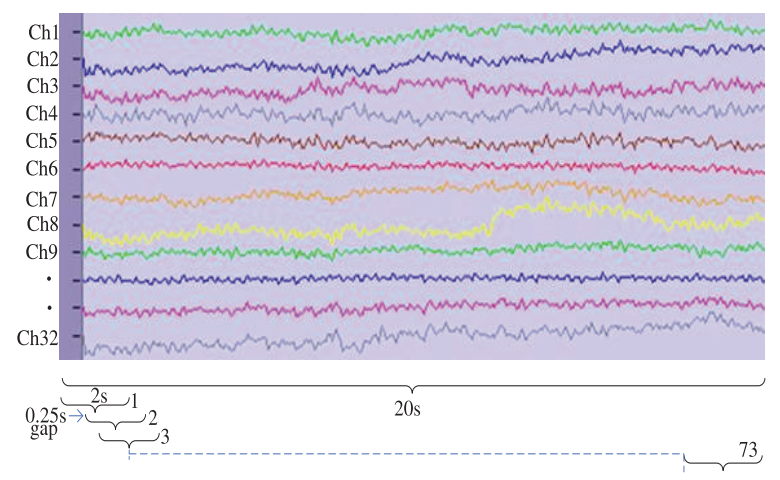

Fig. 3. Section of recorded EEG brain signal

The artifacts were removed from the raw EEG dataset using the second order blind identification (SOBI) and canonical correlation [19]. This is continued by dividing the data into alert and fatigue groups. The first 5 minutes of the data when starting the driving experiment was used for alert ground. For the fatigue group, it used from the last 5 minutes of the data before the experiment was stopped. In each group of data, 20s duration of segment for each state (fatigue and alert states) that had least artefacts was used for further analysis.

A moving window of 2 seconds with overlapping of quarter of seconds was applied to 20 seconds segment which resulted of 73 units of overlapping data. With the total of 5 participants, it resulted of 365 units of dataset for fatigue state and another 365 units for alert state or 730 units for combined dataset. The dataset was divided into training set which taken from 3 participants or 438 units of dataset and testing set from 2 participants or 292 units of dataset. The training set was trained by DBN without needing any feature extraction process and it can be observed that the DBN is able to handle high dimensional un-processed raw EEG data.

To evaluate the effectiveness of proposed method, comparison studies between deep generic model-based SVM (DGM-SVM) and power spectrum density features-based 
SVM (PSD-SVM) are performed and analyzed in Table I. In this experiment, a single layer RBM is good enough for high-level features representation. With the use of trial-error method, the trained RBM with 23 hidden neurons achieve better classification accuracy. During unsupervised training of RBM, the hidden layer is initialized randomly and updated using (3) with learning rate $(\gamma)$ of 0.01 until 500 epoches. As the optimal weights between visible and hidden layers are learned, the reconstruction and the input samples become closer and high-level useful features are obtained.

After the training process, the optimized DGM-SVM achieved improved testing accuracy of $73.29 \%$ with 91.10 $\%$ (Sensitivity) and $55.48 \%$ (Specificity) while PSD features itself gives $61.00 \%, 70.00 \%, 52.00 \%$ of accuracy, sensitivity and specificity. Based on the optimized RBM hidden neurons, there are 23 features extracted by DGM while 121 features are extracted by PSD. It can be seen that the proposed DGM-SVM can effectively perform in detection of driver fatigue with high-level features extracted by DGM.

TABLE I

COMPARISONS STUDIES: BEST TESTING RESULTS

\begin{tabular}{|l|c|c|c|}
\hline METHOD & Sensitivity(\%) & Specificity(\%) & Accuracy(\%) \\
\hline PSD-SVM & 70.00 & 52.00 & 61.00 \\
\hline DGM-SVM & $\underline{\mathbf{9 1 . 1 0}}$ & $\underline{\mathbf{5 5 . 4 8}}$ & $\underline{\mathbf{7 3 . 2 9}}$ \\
\hline
\end{tabular}

Finally, the optimized DGM network structure is constructed by 32 units of input layer (i.e, 32 EEG channels), 23 units of hidden RBM layer and 1 output units of final SVM layer. Since the architecture of network, especially the size of RBM can greatly influence the performance, it is vital to carefully select the number of hidden RBM units and/or layers. There is trade-off between too many and too less number of hidden neurons and/or layers. To the best of knowledge, there is no special techniques proposed for the selection of optimal network parameters.

\section{CONCLUSIONS}

In this paper, by the use of 32 EEG channels, a hybrid deep generic model (DGM)-based SVM is developed for detection of driver fatigue. With the proposed integrated framework, the most abstract features are generated through the layerwise training of RBM deep architectures and applied to SVM for the best classification performances. By fusing the unsupervised feature extraction and SVM classification, the integrated framework becomes stronger and enhances the performance of feature extraction and classification. To evaluate the effectiveness of proposed method, several experiments are conducted and analyzed. With the use of proposed method, the improvement in accuracy, sensitivity and specificity is satisfactorily found. It means that the DGM is useful for extracting high-level features and improved the performance of driver fatigue detection system. In short, the proposed hybrid DGM-based SVM driver fatigue detection system can effectively detect the normal and fatigue stages through analysis of large variation of drivers' EEG signal.

\section{REFERENCES}

[1] G. Matthews, P. A. Hancock, M. C. Neubauer, and P. A. Desmond, The handbook of operator fatigue: Ashgate Publishing, Ltd., 2012.

[2] S. K. L. Lal and A. Craig, "A critical review of the psychophysiology of driver fatigue", Biological Psychology, vol. 55, pp. 173-194, 2001.

[3] J.-E. Lv, L.-H. Zhu, R.-S. Zheng, and Y.-F. Wei, "Effects of driver's reaction time on safe driving". Journal of transportation systems engineering and information technology, vol. 14, pp. 80-86, 2014.

[4] A. Craig, Y. Tran, N. Wijesuriya, and H. Nguyen, "Regional brain wave activity changes associated with fatigue," Psychophysiology, vol. 49, pp. 574-582, 2012.

[5] A. Craig, Y. Tran, N. Wijesuriya, and P. Boord, "A controlled investigation into the psychological determinants of fatigue," Biological Psychology, vol. 72, pp. 78-87, 2006.

[6] S. Hu and G. Zheng, "Driver drowsiness detection with eyelid related parameters by Support Vector Machine," Expert Systems with Applications, vol. 36, pp. 7651-7658, 2009

[7] Y. Tran, N. Wijesuriya, M. Tarvainen, P. Karjalainen, and A. Craig, "The relationship between spectral changes in heart rate variability and fatigue," Journal of Psychophysiology, vol. 23, pp. 143-151, 2009.

[8] I. G. Campbell, "EEG recording and analysis for sleep research,' Current Protocols in Neuroscience, pp. 10.12. 11-10.12. 19, 2009.

[9] G. E. Hinton, "Learning multiple layers of representation", Trends in Cognitive Sciences, vol. 11, no. 10, pp. 428-434, 2007.

[10] H. P. Martinez, Y. Bengio and G. N. Yannakakis, "Learning multiple layers of representation", IEEE Computational Intelligence Magazine, vol. 8, no. 2, pp. 20-33, 2013.

[11] G. E. Dahl, M. Ranzato, A. Momamed and G. E. Hinton, "Phone recognition with the mean-covariance restricted noltzmann machines", Advances in Neural Information Processing Systems of NIPS, 2010.

[12] R. Sarikaya, G. E. Hinton and A. Deoras, "Application of deep belie networks for natural language understanding", IEEE/ACM Transactions on Audio, Speech and Language Processing, no. 22, vol. 4, pp. 778-784, 2014.

[13] D. F. Wulsin, J. R. Gupta and R. Mani, "Journal of Neural Engineering", Modeling electroencephalography waveforms with semisupervised deep belief nets: fast classification and anomaly measurement, vol. 8, no. 3, pp. 1-13, 2011.

[14] H. Lee, R. Grosse and R. Ranganath, "Convolutional deep belief networks for scalable unsupervised learning of hierarchical representations", Proceedings of the 26th Annual International Conference on Machine Learning, vol. 15, pp. 609-616, 2009.

[15] G. E. Hinton, S. Osindero and Y. W. Teh, "A fast learning algorithm for deep belief nets", Neural Computation, vol. 18, no. 7, pp. 1527 1554, 2006.

[16] A. Fischer and C. Igel, "An introduction to rstricted boltzmann machines", Progress in Pattern Recognition, Image Analysis, Computer Vision, and Applications, vol. 7441, pp. 14-36, 2012.

[17] F. J. Huang and Y. LeCun , "Large-scale Learning with SVM and Convolutional for Generic Object Categorization," IEEE Computer Society Conference on Computer Vision and Pattern Recognition, vol. 1, pp. 284-291, 2006.

[18] D. G. Altman and J. M. Bland, "Statistics Notes: Diagnostic tests 1: sensitivity and specificity", Clinical chemistry, vol.308, pp. 1552-1552, 1994.

[19] Y. Tran, A. Craig, N. Wijesuriya, and H. Nguyen, "Improving classification rates for use in fatigue countermeasure devices using brain activity," in Proc. of the 32 Annual International Conference of the IEEE Engineering in Medicine and Biology Society (EMBC), 2010, pp. 4460-4463. 